\title{
Diversity of thermal responses of lipid composition in the membranes of the dominant culturable members of an Antarctic fellfield soil bacterial community
}

\author{
P. LEWIS WHITE', DAVID D.WYNN-WILLIAMS ${ }^{2}$ and NICHOLAS J. RUSSELL * * \\ ${ }^{1}$ Department of Biological Sciences, Wye College, University of London, Wye, Ashford, Kent TN25 5AH, UK \\ ${ }^{2}$ British Antarctic Survey, Natural Environment Research Council, High Cross, Madingley Road, Cambridge CB3 OET, UK \\ *corresponding author-n.russell@wye.ac.uk
}

\begin{abstract}
The eight dominant culturable members of an Antarctic fellfield soil bacterial community were four Arthrobacter species, Sanguibacter suarezil, Aureobacterium testaceum, a Bacillus sp., and a Pseudomonas sp. All of the isolates grew at $2^{\circ} \mathrm{C}$, but two of the Arthrobacter spp. were psychrophilic, while the other six bacterial species were psychrotolerant. However, the fastest growing organisms at low temperatures were not the psychrophiles, and the psychrotolerant Bacillus sp. grew fastest at temperatures up to $25^{\circ} \mathrm{C}$. When the growth temperature of cultures was altered, the phospholipid content of the two psychrophilic Arthrobacter spp. decreased, whereas the phospholipid contents of the psychrotolerant spp. either increased or did not change. Only one psychrophilic and one psychrotolerant Arthrobacter sp. modified its polar lipid head-group composition in response to a lowering of growth temperature. The change in Arthrobacter sp. CL2-1 was particularly marked and novel in that at low temperatures phosphatidylethanolamine was replaced completely by a phosphoglycolipid and phosphatidylserine, neither of which was present at higher growth temperatures. All eight isolates altered the fatty acyl compositions of their membrane lipids in a manner that was only partially dependent on taxonomic status. In Bacillus sp. C2-1 the changes were opposite to that predicted on the basis of membrane fluidity considerations. The isolates used different combinations of changes in fatty acid branching, unsaturation and chain length. There was no single strategy of thermal adaptation that was employed and the variety of strategies used did not follow phylogenetic boundaries.
\end{abstract}

Received 11 November 1999 , accepted 21 February 2000

Key words: Antarctic soil, arthrobacters, fatty acids, membrane lipids, psychrophiles, psychrotolerant bacteria, thermal adaptation

\section{Introduction}

If microorganisms are to colonize cold habitats successfully, they must adapt their cellular compositions because they are too small to insulate themselves. A key feature of such thermal adaptive responses is the alteration in membrane lipid composition in order to provide the correct fluidity and phase which is essential for the maintenance of passive permeability and the activity of membrane proteins, for example those responsible for electron transport and uptake of nutrients (Russell 1989). Such changes in response to temperature are most evident in the fatty acyl composition of membrane phospholipids and glycolipids, although there may also be alterations to the lipid head-group composition. Bacteria respond to a lowering of temperature by increasing the proportion of unsaturated or branched fatty acyl chains, or the relative amount of anteiso- compared to iso-branching, or by shortening the fatty acyl chain of the constituent membrane phospholipids and (phospho)glycolipids (Russell 1984). These changes may occur either alone or in combination.

Bacteria in Antarctic soils have to be able to withstand temperatures from well below zero to $20^{\circ} \mathrm{C}$ or even higher over time-scales ranging from seasonal to diurnal or shorter.
Therefore, they might be expected to exhibit particularly welldefined adaptive responses of their membrane lipid composition. Although there have been many studies of the effects of temperature changes on membrane lipid fatty acyl composition, including those in bacteria isolated from Antarctic soils, they have generally been of single isolates. No coordinated effort has been made to compare how the members of a microbial community within a defined habitat respond to environmental shifts in temperature. In this paper we present the results of an investigation of the lipid composition of the dominant culturable members of a bacterial community containing a number of psychrophilic and psychrotolerant bacteria isolated from a fellfield soil on Signy Island, in the Maritime Antarctic. It is not possible, of course, to study the lipid composition of non-culturable members of the community. We show that there is no single response of fatty acid composition that is favoured above another: instead, a diverse range of responses are utilized that may cross phylogenetic boundaries. 
Table I. Phenotypic characteristics of selected Antarctic bacterial isolates

\begin{tabular}{|c|c|c|c|c|c|}
\hline Strain & Gram reaction & Colony colour & Shape & Motility & Arrangement \\
\hline Arthrobacter sp. Cl-1 & + & yellow & ovoid/coccoid & - & single cells \\
\hline Arthrobacter sp.C2-1a & + & yellow & ovoid/coccoid & - & chains \\
\hline Arthrobacter sp. C2-2 & + & creamy/grey & ovoid & - & chains \\
\hline Arthrobacter sp.CL2-1 & + & white & ovoid & - & pairs/tetrads \\
\hline Sanguibacter suarezii & + & yellow & ovoid & - & chains \\
\hline Aureobacterium testaceum & + & orange & ovoid & - & pairs/tetrads \\
\hline Pseudomonas sp. CL1-1 & - & pale yellow & bacilloid & $+/-$ & single cells \\
\hline Bacillus sp. C2-1 & + & white & bacilloid & $+/-$ & single cells \\
\hline
\end{tabular}

\section{Materials and methods}

Source of bacteria

The bacteria were isolated from fellfield soil samples collected in 1995 from frost-sorted polygons at Jane Col, Signy Island $\left(60^{\circ} 43^{\prime} \mathrm{S}, 45^{\circ} 36^{\prime} \mathrm{W}\right)$. The soil samples $(c .10 \mathrm{~g})$ were maintained frozen at $-20^{\circ} \mathrm{C}$ from the time of collection until being thawed at room temperature. The soil samples were suspended in $0.1 \mathrm{M}$ sodium phosphate buffer (c. $0.1 \mathrm{mg}$ soil ml-1 buffer), shaken vigorously by hand and allowed to settle. Aliquots $(0.5 \mathrm{ml})$ were plated on nutrient agar and incubated at $5^{\circ} \mathrm{C}$ for periods up to three weeks. Plates were examined at intervals and individual colonies picked off and replated on fresh nutrient agar until judged pure by colonial morphology and by cellular morphology using transmitted light microscopy.

\section{Bacterial identification}

Individual isolates were identified initially on the basis of their Gram reaction, colonial and cellular morphology (light microscopy) and their biochemical characteristics using the API $20 \mathrm{NE}$ and $50 \mathrm{CHB}$ test kits (bioMérieux). The test kits were used according to the manufacturer's instructions except that the incubation temperature was $20^{\circ} \mathrm{C}$ (instead of $37^{\circ} \mathrm{C}$ ) and incubations were continued for up to four days in order to ensure that end-points of the indicator reactions had been reached at the lower temperature. Catalase and peroxidase activities were also tested independently. Putative identities were checked by fatty acid analysis of total cellular lipids using capillary-gas-liquid chromatography and confirmed by

Table II. Cardinal growth temperatures of selected Antarctic bacterial isolates.

\begin{tabular}{lcccl}
\hline \multirow{5}{*}{ Strain } & Cardinal growth temperatures $\left({ }^{\circ} \mathrm{C}\right)$ \\
& Optimum & Min & Max & Type \\
\hline Arthrobacter sp. C1-1 & $12-15$ & $<2$ & 21 & psychrophilic \\
Arthrobacter sp. C2-1 a & 15 & 2 & 19 & psychrophilic \\
Arthrobacter sp. C2-2 & $20-22$ & $<2$ & 33 & psychrotolerant \\
Arthrobacter sp. CL2-1 & 25 & 2 & 31 & psychrotolerant \\
Sanguibacter suarezii & $25-28$ & 2 & 33 & psychrotolerant \\
Aureobacterium testaceum & 28 & 3 & 36 & psychrotolerant \\
Pseudomonas sp. CL1-1 & 28 & $3-4$ & 36 & psychrotolerant \\
Bacillus sp. C2-1 & 28 & 2 & 40 & psychrotolerant \\
\hline
\end{tabular}

16S rDNA analysis.

Total polar lipids were extracted using a modified Bligh \& Dyer method as described by Kates (1986). Phospholipids were separated by thin-layer chromatography on plates of silica gel $60 \mathrm{H}$ using a solvent system of chloroform/methanol/ acetic acid/water (85/15/10/3.5, by vol.) and were identified using specific spray reagents (Kates 1986). Phospholipids were quantitated on the basis of lipid phosphorus analysis (Kates 1986). Fatty acids were analysed as methyl ester derivatives formed by transmethylation of acyl lipids using $2.5 \%(\mathrm{v} / \mathrm{v})$ sulphuric acid in dry methanol (Kates 1986). They were analysed by capillary gas chromatography using a Hewlett Packard 5880A gas chromatograph fitted with a Supelco 2380 polar fused silica capillary column and Spectraphysics SP420 integrator. The carrier gas was nitrogen with a flow rate of

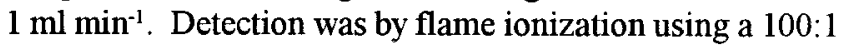
splitter ratio and detector and injector temperatures of $250^{\circ} \mathrm{C}$. The oven temperature was set initially at $130^{\circ} \mathrm{C}$ for $10 \mathrm{~min}$, then ramped to $250^{\circ} \mathrm{C}$ at $4 \mathrm{Celsius} \mathrm{degrees} \mathrm{min}^{-1}$ and held at the final temperature for $15 \mathrm{~min}$. Individual peaks were identified by comparison of their retention times with those of authentic standards, and confirmed by sequential hydrogenation and bromination (Kates 1986).

The sequence of $16 \mathrm{~S}$ rDNA genes was determined by analysis of the PCR products of amplification of genomic DNA using two pairs of primers that are specific for the two variable regions of the gene. The primers were A (5'-GGA GAG TTA GAT CTT GGC TCA G-3') and B (5'-CCA GTG TGG CCG GTC GCC CTC TC-3'); and X (5'-CGT GCC AGC CGC GGT AAT AC-3') and Y (5'-TTG GCC AGC CGC GGT AAT AC-3'). Amplifications were performed with Taq DNA polymerase using a Perkin Elmer GeneAmp PCR system 9600 . Sequences were analysed using a Perkin Elmer ABI Prism 310 genetic analyser. For the taxonomic assignment of isolates, the $16 \mathrm{~S}$ rDNA sequences were assembled using the MegAlign, Editseq and Seqman software with a Power Macintosh computer. Similarity searches were carried out against the NCBI database using the Basic Alignment Search Tool (Blast) programme. The percentage similarity between the new isolates and selected described species was calculated using the Phylip programme of the Wisconsin GCG computer package (1996) version 9. Alignment of the sequences was carried out using the MegAlign 
Table III. Thermal dependence of growth rates of selected Antarctic bacterial isolates in liquid culture.

\begin{tabular}{|c|c|c|c|c|c|c|}
\hline \multirow[b]{2}{*}{ Strain } & \multicolumn{3}{|c|}{ Doubling time (h) } & \multicolumn{3}{|c|}{ Specific growth rate $\left(\mathrm{h}^{-1}\right)$} \\
\hline & $5^{\circ} \mathrm{C}$ & $15^{\circ} \mathrm{C}$ & $25^{\circ} \mathrm{C}$ & $5^{\circ} \mathrm{C}$ & $15^{\circ} \mathrm{C}$ & $25^{\circ} \mathrm{C}$ \\
\hline Arthrobacter sp. C1-1 & 12.2 & 3.4 & $\mathrm{ng}$ & 0.055 & 0.201 & ng \\
\hline Arthrobacter sp. C2-1 a & 16.0 & 3.9 & ng & 0.042 & 0.180 & ng \\
\hline Arthrobacter sp. C2-2 & 14.2 & 3.2 & 2.7 & 0.049 & 0.215 & 0.256 \\
\hline Arthrobacter sp. CL2-1 & nd & 6.4 & 5.4 & nd & 0.111 & 0.125 \\
\hline Sanguibacter suarezii & 24.0 & 7.0 & 6.0 & 0.028 & 0.097 & 0.118 \\
\hline Aureobacterium testaceum & 20.0 & 6.8 & 2.5 & 0.035 & 0.097 & 0.118 \\
\hline Pseudomonas sp. CL1-1 & 18.4 & 4.7 & 2.1 & 0.035 & 0.146 & 0.333 \\
\hline Bacillus sp. C2-1 & 4.0 & 3.9 & 2.0 & 0.173 & 0.180 & 0.347 \\
\hline
\end{tabular}

Abbreviations: $\mathrm{ng}=$ no growth, nd $=$ not determined.

sequence database tools.

\section{Thermal characteristics of growth}

Estimates of the cardinal growth temperatures (i.e. minimum, optimum and maximum) of isolates on plates of nutrient agar were determined using a vertical temperature gradient incubator over the temperature range $2-40^{\circ} \mathrm{C}$ with $c$. 2.5 Celsius degree steps between the shelves of the incubator. The time taken for the appearance of colonies visible to the naked eye was used as the measure of growth. A number of other solid media were also used, including rich and minimal media, but there were no statistically significant ( $95 \%$ confidence limit) differences in

Table IV. Polar lipid composition of selected Antarctic bacterial isolates grown at $15^{\circ} \mathrm{C}$.

\begin{tabular}{lcccccccc}
\hline $\begin{array}{l}\text { Lipid } \\
\text { component }\end{array}$ & C2-1a & C1-1 & CL2-1 & C2-2 & 5C2-1 & CL2-2 & CL1-1 & C2-1 \\
\hline GL 1 & - & - & + & - & + & - & - & - \\
LPG & + & + & - & + & + & + & + & - \\
LPE & - & - & - & - & - & - & + & + \\
GL 2 & + & + & + & + & - & + & - & - \\
PS & - & - & + & - & - & - & - & + \\
PG & +++ & +++ & +++ & ++ & +++ & +++ & ++ & ++ \\
GL 3 & + & - & - & - & - & + & - & - \\
PGL & - & ++ & ++ & + & ++ & - & - & - \\
PE & - & - & ++ & - & - & - & +++ & +++ \\
GL 4 & - & + & - & - & + & + & + & - \\
DPG & ++ & ++ & ++ & ++ & ++ & ++ & ++ & + \\
GL 5 & + & - & + & + & + & - & + & + - \\
\hline
\end{tabular}

GL 1 = glycolipid 1, GL 2 = glycolipid 2, GL 3 = glycolipid 3, GL $4=$ glycolipid 4, GL 5 = glycolipid 5, LPG = lysophosphophatidylglycerol, $\mathrm{LPE}=$ lysophosphatidylethanolamine, $\mathrm{PS}=$ phosphatidylserine, $\mathrm{PG}=$ phosphatidylglycerol, $\mathrm{PE}=$ phosphatidylethanolamine, $\mathrm{PGL}=$

phosphoglycolipid, DPG $=$ diphosphatidylglycerol, +++ to $+/-=$ intensity of lipid staining, - = lipid is absent.

\footnotetext{
${ }^{a}$ The lipid components are listed in order of their $R_{f}$ values on thin-layer chromatography plates developed in a solvent system of chloroform/ methanol/acetic acid/water (85: $15: 3.5: 1$, by vol.). Note that it is not known if PGL or GL-1, GL-2 etc. have the same structure in all isolates in which they occur

${ }^{\mathrm{b}}$ In Arthrobacter sp.CL2-1, PE is absent when grown at 5 or $15^{\circ} \mathrm{C}$, whereas PGL and PS are absent when grown at $25^{\circ} \mathrm{C}$

'Sanguibacter suarezii

dAureobacterium testaceum
}

the cardinal growth temperatures.

More accurate growth rates were determined from optical density (500-600 $\mathrm{nm}$ depending on the isolate) readings of growth in nutrient broth. Individual isolates were grown in $400 \mathrm{ml}$ of medium in 1-litre conical flasks shaken at 150 r.p.m. in a temperature-controlled orbital incubator (New Brunswick, Innova 4330).

\section{Results}

The soil samples yielded eight major bacterial isolates based on the frequency of particular colonial morphotypes on solid media and the gross phenotypic characteristics of cellular shape, arrangement and Gram status. These characteristics are summarized in Table I. Of the eight isolates, seven were Gram-positive and five were pigmented yellow and a sixth was orange. The isolates were identified to the genus level on the basis of their phenotypic characteristics, including fatty acyl composition of polar lipids, and by determination of the sequence of two variable regions of $16 \mathrm{~S} \mathrm{rDNA}$. Two isolates, Sanguibacter suarezii and Aureobacterium testaceum, could also be assigned to a species. The Bacillus sp. $\mathrm{C} 2-1$ and Pseudomonas sp. CLl-1, were weakly motile.

Determination of the cardinal growth temperatures of the isolates revealed that all could grow at low temperatures and that six were psychrotolerant and two psychrophilic (Table II). Of the two psychrophilic arthrobacters, only Arthrobacter sp. C2-1a was strictly psychrophilic according to the exact definition of Morita (1975); the other, Arthrobacter sp. C1-1, although its optimum growth temperature was lower than that of Arthrobacter sp. C2-1a, had an upper limit of $21^{\circ} \mathrm{C}$ which is above the value of $20^{\circ} \mathrm{C}$ as defined by Morita. One of the psychrotolerant isolates, Arthrobacter sp. C2-2, had a lower minimum growth temperature (below $2^{\circ} \mathrm{C}$ ) compared to the others. The psychrotolerant Bacillus $\mathrm{C} 2-1$ had a particularly wide growth temperature range of almost 40 Celsius degrees.

The rates of growth in liquid medium at three representative temperatures are summarized in Table III, which confirmed that the two psychrophiles were unable to grow at $25^{\circ} \mathrm{C}$. Among the psychrotolerant isolates, the strain with the lowest optimum temperature, Arthrobacter sp. C2-2, did not have the fastest growth rate at $5^{\circ} \mathrm{C}$; instead it was Bacillus $\mathrm{C} 2-1$, which 
Table V. The effect of growth temperature on phospholipid content of selected Antarctic bacteria.

\begin{tabular}{|c|c|c|c|}
\hline \multirow[b]{2}{*}{ Strain } & \multicolumn{3}{|c|}{ Phospholipid content $\mathrm{mg} \mathrm{g}^{-1}$ cells (dry weight) } \\
\hline & $5^{\circ} \mathrm{C}$ & $15^{\circ} \mathrm{C}$ & $25^{\circ} \mathrm{C}$ \\
\hline Arthrobacter sp. C1-1 & $46.4 \pm 1.8$ & $61.5 \bullet 1.1$ & ng \\
\hline Arthrobacter sp. C2-1 a & $34.9 \pm 2.3$ & $59.0 \pm 1.7$ & ng \\
\hline Arthrobacter sp. C2-2 & $38.8 \pm 2.6$ & $42.1 \pm 2.2$ & $41.5 \pm 1.8$ \\
\hline Arthrobacter sp. CL2-1 & $60.1 \pm 2.3$ & $52.9 \pm 1.0$ & $40.1 \pm 1.4$ \\
\hline Sanguibacter suarezii & $41.7 \pm 2.4$ & $38.4 \pm 2.0$ & $45.9 \pm 1.8$ \\
\hline Aureobacterium testaceum & $74.8 \pm 3.5$ & $71.6 \pm 2.7$ & $71.9 \pm 2.2$ \\
\hline Pseudomonas sp. CL1-1 & $48.9 \pm 1.3$ & $42.5 \pm 1.1$ & $34.2 \pm 2.5$ \\
\hline Bacillus sp. C2-1 & $50.7 \pm 2.1$ & $40.5 \pm 1.3$ & $31.8 \pm 1.9$ \\
\hline
\end{tabular}

$\mathrm{ng}=$ no growth.

Data values are the means $\pm \mathrm{s} d$ of analyses of three independent cultures.

not only grew 3-6 times faster at $5^{\circ} \mathrm{C}$ than did the other psychrotolerant strains, but also grew at the same rate at $15^{\circ} \mathrm{C}$ and two-fold faster at $25^{\circ} \mathrm{C}$.

The polar lipid compositions of the eight isolates grown at $15^{\circ} \mathrm{C}$ are given in Table IV. The seven Gram-positive isolates had compositions that were typical of their genus, in that phosphatidylglycerol (PG) was the major lipid together with diphosphatidylglycerol (DPG); typically for bacilli, Bacillus sp. C2-1 also contained phosphatidylethanolamine (PE) as a major polar lipid. The Gram-positive isolates all contained 1-3 glycolipids and four isolates also contained a phosphoglycolipid. Although their structures were not determined, there were at least five different glycolipids and one phosphoglycolipid, based on their retention times on thinlayer chromatography, and it was possible, for instance, to distinguish the four arthrobacters on the basis of different (phospho)glycolipid profiles (Table IV). The Gram-negative Pseudomonas sp. CL1-1 also had a composition that was typical of its genus, with PE predominating together with PG and DPG.

When the temperature of the two psychrophilic isolates Arthrobactersp. C1-1 and C2-1 a was lowered from 15 to $5^{\circ} \mathrm{C}$, the phospholipid content decreased by 25 and $40 \%$ respectively (Table V). The phospholipid contents of the psychrotolerant isolates either increased by up to $33 \%$ or did not change.

When cultures were grown at temperatures between 5 and $25^{\circ} \mathrm{C}$, no statistically-significant (confidence level $=95 \%$ ) differences in polar lipid compositions were seen, except for
Table VI. The effect of growth temperature on polar lipid fatty acid composition (wt \%) of Arthrobacter sp. C1-1.

\begin{tabular}{lcccccccc}
\hline & \multicolumn{2}{c}{ Total polar lipid } & \multicolumn{2}{c}{ PGL } & \multicolumn{2}{c}{ PG } & \multicolumn{2}{c}{ DPG } \\
Fatty acid & 5 & 15 & 5 & 15 & 5 & 15 & 5 & 15 \\
\hline$a 15: 0$ & 67.0 & 56.6 & 72.1 & 66.7 & 57.7 & 34.5 & 8.3 & 7.3 \\
n15:0 & 8.9 & 4.7 & 6.5 & 5.3 & 4.4 & tr & 5.8 & 3.8 \\
$i 16: 0$ & 4.7 & 3.3 & 3.0 & 7.5 & 4.4 & 21.2 & 17.8 & 18.3 \\
a16:0 & 2.3 & tr & 3.7 & tr & nd & nd & 12.1 & 10.8 \\
$a 17: 0$ & 14.2 & 20.7 & 14.7 & 15.2 & 32.5 & 41.0 & 42.0 & 42.2 \\
n17:0 & 2.0 & 10.1 & tr & 5.3 & 1.1 & 3.2 & 14.0 & 17.6 \\
ratio a/i & 15.7 & 11.2 & 29.2 & 10.9 & 20.5 & 3.6 & 3.5 & 3.3 \\
ratio br/n & 8.0 & 5.4 & 14.9 & 8.1 & 17.5 & 29.3 & 4.1 & 3.6 \\
ACL & 16.03 & 16.73 & 15.36 & 15.47 & 15.71 & 16.08 & 16.42 & 16.49 \\
\hline
\end{tabular}

$\mathrm{PGL}=$ phosphoglycolipid, $\mathrm{PG}=$ phosphatidylglycerol,

$\mathrm{DPG}=$ diphosphatidylglycerol, $a=$ anteiso-branched, $\mathrm{n}=$ straight chain, $i=$ iso- branched, $\mathrm{br}=$ total branched (anteiso\& iso), $\mathrm{ACL}=$ average chain length, $\operatorname{tr}=$ trace $(<0.5 \%)$, nd $=$ not detected.

Arthrobacter spp. C2-2 and CL2-1. In Arthrobacter sp. C2-2 there was a $12 \%$ decrease in the proportion of $P G$ and increases of 4 and $6 \%$ respectively in lyso-PG and DPG when the growth temperature was lowered to $5^{\circ} \mathrm{C}$ (data not shown). The DPG is derived metabolically from PG and the lyso-PG may be a degradation product or a metabolic intermediate related to PG metabolism (Harwood \& Russell 1984). When Arthrobacter sp. CL2-1 was grown at its optimum growth temperature of $25^{\circ} \mathrm{C}$ (Table II), it contained PG as the major polar lipid (62\%), together with $25 \% \mathrm{PE}$ and $13 \%$ DPG and small amounts of a glycolipid (data not shown). In contrast, when grown at 5 or $15^{\circ} \mathrm{C}$, although the major polar lipid was still PG (51-52\%) together with DPG (11-14\%) and a small amount of glycolipid, the PE was replaced completely by a phosphoglycolipid (25\%) and phosphatidylserine (10-11\%), neither of which was present at the higher temperature.

The fatty acyl compositions of the polar lipids in all eight isolates were found to be dependent on the culture temperature. For example, the psychrophilic Arthrobacter sp. C1-1 had a complex fatty acid composition, typical for the genus, comprised of branched and straight-chain fatty acids with odd and even-numbered carbon chains, and responded to a decrease in growth temperature by increasing the proportion of branched chains with a higher ratio of anteiso- to iso-branches and a shorter average chain length (Table VI). In contrast, the

Table VII. Summary of thermal adaptations in fatty acid composition of selected Antarctic bacteria in response to lower growth temperature.

\begin{tabular}{|c|c|c|c|c|}
\hline Strain & Branched relative to straight chain & Anteiso-relative to iso-branched & Average chain length & Unsaturation \\
\hline Arthrobacter sp. C1-1 & $\boldsymbol{\uparrow}$ & $\boldsymbol{\uparrow}$ & $\downarrow$ & n.r. \\
\hline Arthrobacter sp. C2-1a & $\boldsymbol{\uparrow}$ & $\uparrow$ & n.c. & n.r. \\
\hline Arthrobacter sp. C2-2 & $\uparrow$ & $\uparrow$ & $\downarrow$ & n.r. \\
\hline Arthrobacter sp. CL2-1 & $\uparrow$ & $\boldsymbol{r}$ & n.c. & $\uparrow$ \\
\hline Sanguibacter suarezii & $\downarrow$ & $\boldsymbol{T}$ & $\downarrow$ & n.r \\
\hline Aureobacterium testaceum & $\uparrow$ & $\uparrow$ & $\downarrow$ & n.r. \\
\hline$P_{\text {seudomonas sp. } \mathrm{CLI}-1}$ & n.r. & n.r. & n.c. & $\uparrow$ \\
\hline Bacillus sp. C2-1 & $\downarrow$ & $\downarrow$ & n.c. & n.c. \\
\hline
\end{tabular}

n.r. $=$ not relevant (because the relevant fatty acids are not present),n.c. $=$ no significant change. 
psychrotolerant Arthrobacter sp. C2-2 had a very similar fatty acid composition, both qualitatively and quantitatively (data not shown) and yet it responded to a decrease in temperature by altering its fatty acid composition in the same manner, despite having very different cardinal temperatures to Arthrobacter sp. C1-1 (Table II). The data in Table VI also illustrate the fact that the temperature-dependent changes in fatty acid composition observed for the total polar lipid fraction were not reflected uniformly in all of the individual lipid components, which not only had different fatty acid compositions but also responded differently to temperature changes. This effect was noted for several of the isolates (data not shown). Arthrobacter sp. CL2-1 had a different fatty acid composition to the other three Arthrobacter spp., in that it contained $16: 1$ in addition to the usual branched-chain components (cf. Table VI composition for Arthrobacter sp. $\mathrm{Cl} 1$ 1) when grown at low temperatures; at $5^{\circ} \mathrm{C} 16: 1$ was $11 \%$ of the total fatty, decreasing to $6 \%$ at $15^{\circ} \mathrm{C}$, whereas at $25^{\circ} \mathrm{C}$ (its optimum growth temperature) there was none of this component (data not shown). Arthrobacter sp. CL2-1 also increased the proportion of branched fatty acids and their ratio of anteiso- relative to iso-branching at lower growth temperatures, as observed for the other three arthrobacters. Arthrobacter spp. C1-1 and C2-2 decreased the average chain length of the fatty acids at lower temperatures, whereas Arthrobacter spp. C2-1a and CL2-1 did not.

The temperature-dependent fatty acid compositional changes for all eight isolates are summarized in Table VII, which shows that a variety of modifications occurred amongst the seven Gram-positive isolates in response to a decrease in culture temperature. All of the changes, apart from those for Bacillus sp. C2-1, would serve to maintain membrane fluidity at lowered growth temperatures. This isolate was unusual in that it displayed only very small temperature-dependent changes in fatty acid branching and chain length, yet all the parameters were the opposite of that which would be predicted on the basis of fluidity considerations (Table VIII). The apparently anomalous decrease in proportion of branched fatty acids in Sanguibacter suarezii at lower temperatures was small compared to the larger (fluidizing) changes in proportion of anteiso relative to iso-branched fatty acids of shorter average chain length (Table VII).
Table VIII. The effect of growth temperature on polar lipid fatty acid composition (wt \%) of Bacillus sp. C2 -1 .

\begin{tabular}{lrrr}
\hline \multicolumn{3}{c}{ Growth temperature $\left({ }^{\circ} \mathrm{C}\right)$} \\
Fatty acid & 5 & 15 & 25 \\
\hline$a 14: 0$ & 1.0 & 1.1 & 2.0 \\
$i 15: 0$ & 2.9 & 5.7 & 8.4 \\
$a 15: 0$ & 48.7 & 55.8 & 62.7 \\
$i 16: 0$ & 2.5 & 3.0 & 3.4 \\
$a 16: 0$ & 7.9 & 4.9 & 1.8 \\
n16:0 & 4.6 & 5.1 & 5.1 \\
$16: 1$ & 3.5 & 4.2 & 4.8 \\
$i 17: 0$ & 17.1 & 11.3 & 5.3 \\
n17:0 & 7.8 & 6.9 & 2.5 \\
Ratio $a / i$ & 2.6 & 3.1 & 3.9 \\
Ratio br/n & 4.5 & 4.5 & 5.1 \\
ACL & 15.5 & 15.6 & 15.0 \\
\hline
\end{tabular}

Abbreviations: see Table VI.

The Gram-negative Pseudomonas sp. CL1-1 had a fatty acid composition dominated by even-carbon-numbered saturated and monounsaturated components, and responded to a decrease in growth temperature by increasing the proportion of unsaturated fatty acids, a change that was reflected in the individual major polar lipids (Table IX).

\section{Discussion}

The preponderance of Gram-positive isolates among the eight dominant culturable members of the soil community was unexpected because generally it has been found that Gramnegative bacteria predominate in maritime Antarctic soils (Wynn-Williams 1990, Friedmann 1993). However, as part of another study, we have analysed larger numbers of bacteria from the same Signy fellfield soils (up to 80 per soil sample) that form as colonies on initial isolation plates and have found that $58-67 \%$ are Gram-negative (White 1999). The eight isolates studied here represent those which predominated on plates of nutrient agar, but we have not found that changing the medium either to another rich (broth-based) one, at full strength or diluted, or using a defined minimal medium, alters the pattern of dominant organisms obtained. It is recognized that these represent a fraction of the culturable bacteria present and an even smaller proportion of the total numbers

Table IX. The effect of growth temperature on polar lipid fatty acid composition (wt \%) of Pseidomonas sp. CL1-1.

\begin{tabular}{|c|c|c|c|c|c|c|c|c|c|}
\hline \multirow[b]{2}{*}{ Fatty acid ${ }^{\mathbf{a}}$} & \multicolumn{3}{|c|}{ Total polar lipid } & \multicolumn{2}{|c|}{$\mathrm{PE}$} & \multicolumn{2}{|c|}{ PG } & \multicolumn{2}{|c|}{$\mathrm{DPG}$} \\
\hline & 5 & 15 & 25 & 5 & 15 & 5 & 15 & 5 & 15 \\
\hline $16: 0$ & 18.1 & 27.8 & 30.3 & 25.0 & 24.3 & 10.8 & 28.6 & 19.5 & 10.7 \\
\hline $16: 1$ & 48.5 & 47.9 & 36.2 & 43.0 & 45.4 & 21.9 & 11.0 & 7.6 & 32.8 \\
\hline $17: 0$ & 3.3 & 3.6 & 8.6 & 1.4 & 3.5 & 17.1 & 7.5 & 20.4 & 6.2 \\
\hline $18: 0$ & 4.0 & 3.9 & 15.0 & nd & nd & 8.1 & 13.2 & 6.4 & 6.0 \\
\hline $18: 1$ & 26.1 & 16.1 & 9.2 & 25.3 & 12.8 & 24.3 & 8.9 & 49.3 & 13.0 \\
\hline UI & 0.75 & 0.64 & 0.45 & 0.70 & 0.59 & 0.46 & 0.20 & 0.65 & 0.49 \\
\hline $\mathrm{ACL}$ & 16.73 & 16.39 & 16.50 & 16.13 & 16.37 & 16.60 & 16.60 & 16.90 & 16.30 \\
\hline
\end{tabular}

$\mathrm{UI}=$ unsaturation index; and see Table VI.

${ }^{a}$ All fatty acid components are straight chain. 
present, most of which (i.e. probably $>99 \%$ ) will be unculturable (Torsvik et al. 1996). Nonetheless, it is a truism that one can only perform biochemical analyses of such parameters as lipid composition and measure bulk physiological responses on those organisms which can be cultivated.

Arthrobacters, which represented half of the dominant isolates, are commonly found in cold habitats. The four isolates are distinct from each other on the basis of phenotypic and molecular (16S rDNA) characteristics. They are similar to Arthrobacter polychromogenes, but none has greater than $98.5 \%$ similarity for either variable region of the $16 \mathrm{~S} \mathrm{rRNA}$, and all can be distinguished from $A$. polychromogenes on the basis of phenotypic characteristics. A more detailed phylogenetic study is required using molecular techniques in order to assign them to species.

All of the isolates were able to grow at a temperature of $2^{\circ} \mathrm{C}$, although there was no correlation between cardinal growth temperatures and whether the organism is psychrotolerant or psychrophilic strictly according to the definition of Morita (1975). The psychrophilic Arthrobacter sp. C1-1 and the psychrotolerant Arthrobacter sp. C2-2 were both able to grow below this temperature, but the two psychrophilic arthrobacters have lower optimum growth temperatures compared with all the psychrotolerant isolates, whether arthrobacters or not. The predominance of psychrotolerant isolates is a common finding for even permanently cold environments and, although the temperature of most Antarctic soils seldom rises above $10^{\circ} \mathrm{C}$, exposed soils may reach temperatures as high as $20-25^{\circ} \mathrm{C}$ during the summer. Therefore, the psychrotolerant isolates with growth optima at $20-28^{\circ} \mathrm{C}$ are able to take full advantage of the short time when the soil is warmed. The soil samples were collected during summer, which could account for predominance of psychrotolerant bacteria in the laboratory isolations. With their wide growth temperature ranges of 31-38 Celsius degrees combined with the ability to grow at low temperatures, the psychrotolerant bacteria are likely to be able to compete effectively with the psychrophiles, particularly because they have growth rates which are as fast as or faster than those of the psychrophiles (cf. Tables II \& III). The Bacillus sp. C2-1 is noteworthy for both its very wide growth temperature range of 38 Celsius degrees and fast growth rates; in addition, it produces extracellular polysaccharide (White 1999), which could act as a protectant against transient desiccation as a result of exposure to winds or freezing alternating with heavy precipitation. Changeable weather is a feature of the maritime Antarctic fellfields.

The polar lipid compositions are essentially typical of the taxonomic status of the bacteria, although there is little comparative data for species of Aureobacterium and Sanguibacterium. Only two isolates changed their polar lipid composition in response to shifts in growth temperature, which agrees with published reports (e.g. see Bhakoo \& Herbert 1980 and discussion in Russell 1990). Moreover, in the present study, the changes in lipid composition of Arthrobacter sp. C2-2 may reflect alterations in growth rate rather than growth temperature, since the organism grows five-fold slower at $5^{\circ} \mathrm{C}$ compared with $25^{\circ} \mathrm{C}$. Nonetheless, the lipid composition changes are consistent with being an adaptation for low temperature growth, since the increase in DPG content from $2 \%$ at $25^{\circ} \mathrm{C}$ to $8 \%$ at $5^{\circ} \mathrm{C}$, largely at the expense of $P G$, would lower the liquid-crystalline to gel phase transition of the membrane lipids (Boggs et al. 1981, Russell 1989).

The large qualitative changes in Arthrobacter sp. CL2-1, however, are certainly not related to growth rate because the bacterium has quite a different lipid composition at 15 and $25^{\circ} \mathrm{C}$ when the growth rates are very similar. Moreover, such a radical temperature-dependent switch between two different lipid compositions has not been reported previously. The change in lipid composition will affect such fundamental properties as the charge distribution and packing of the lipids in the membrane. It is reversible, occurring rapidly after either a shift-up or shift-down in temperature (White 1999), the details of which will be published separately.

The psychrophilic isolates adapt to a lower temperature by reducing their lipid content, whereas the psychrotolerant isolates either do not change or increase the total amount of lipid in their membranes. This distinction is not a reflection of taxonomic status, because the psychrophilic arthrobacters behave differently from the psychrotolerant arthrobacters. An increase in lipid content with a lowering of temperature has been observed previously (Wada et al. 1987) and it has been postulated that this may be a mechanism for increasing membrane area to incorporate extra proteins for nutrient uptake, thus enhancing the chance of survival in the cold (Russell 1990). Conversely, it has been argued that for photosynthetic microorganisms smaller cells will have more efficient photosynthesis because of the closer packing of pigments and therefore more effective energy transfer (Vincent 1988). Among the psychrotolerant isolates, the three which maintained a constant lipid composition across their growth temperature range (Arthrobacter sp. C2-2, Sanguibacter suarezii and Aureobacterium testaceum) are all pigmented. Although these bacteria are not photosynthetic, retention of a close packing of pigments such as carotenoids in their membranes may be a mechanism for protecting against UV radiation. The psychrotolerant Arthrobacter sp. CL2-1, which does modify its lipid content with changes of temperature, is not pigmented (cf. Tables I \& V).

A major aim of this study was to determine whether there are any general strategies of membrane fatty acid changes in Antarctic bacteria in response to alterations in growth temperature that might be related to whether the organisms are psychrophilic or psychrotolerant. The dominant community members all have fatty acyl compositions of their polar lipids that reflect their taxonomic status, yet they display a diversity of responses to temperature change. Members of the same genus, even if they have very similar fatty acid compositions, may use a different strategy to modify fatty acid composition and thereby maintain membrane fluidity and function. For 
example, among the four Arthrobacter spp., three have fatty acid compositions which are typical of the genus, but Arthrobacter sp. CL2-1 contains 16:1 in addition, and three different strategies of fatty acid adaptations are adopted by the arthrobacters.

Among the eight dominant members of the soil community, six different strategies are used, as summarized in Table VII. However, that summary hides the fact that individual lipids may not only have different fatty acid compositions but also respond differently to a shift in bacterial growth temperature. For example, the psychrophilic Arthrobactersp. Cl-l modifies its fatty acid composition at lower temperatures by shortening the average chain length, increasing the proportions of branched-chain fatty acids and increasing the ratio of anteisoto iso-branching, similar to the adaptations noted in the psychrophilic Arthrobacterglacialis by Canillac et al. (1982). The main alterations at lower temperatures in Arthrobacter sp. C1-1 are increases in $n 15: 0$ and $a 15: 0$ relative to $i 15: 0$, whilst the change in branching ratio is small compared to the large proportion of branched fatty acids present. In contrast, the psychrophilic Arthrobacter sp. C2-1a, which has a similar fatty acid composition to the other psychrophilic Arthrobacter sp. Cl-1, adapts differently to lower temperatures by increasing branched fatty acids, particularly relative to straight-chain fatty acids, and increasing anteiso-relative to iso-branching. However, the average chain length does not alter because there are changes in a 17:0 which do not occur in Arthrobacter sp. C1-1.

In Arthrobacter sp. C2-1a the fatty acid changes are observed in all of the lipid components, with the greatest alteration being in DPG, whereas in Arthrobacter sp. Cl-1 the changes are smaller in DPG compared to the other lipids. All of the lipids in Arthrobacter sp. C2-la respond by increasing the proportion of anteiso-branching at lower temperatures and all lipids exceptDPGalso increase the proportion of total branched fatty acids, whereas in Arthrobacter sp. C1-1 there is a shortening of the fatty acyl chain length and little change in fatty acid branching in DPG. Therefore, although the two arthrobacters are genotypically and phenotypically very similar, they have developed independent mechanisms of fatty acid modification to lower growth temperatures.

The thermal adaptation of fatty acid composition in Bacillus sp. C2-1 is different to all of the other isolates. The major alteration is a decrease in anteiso-relative to iso-branching, due to the introduction of $i 17: 0$ at the expense of $a 15: 0$ (Table VIII). This is apparently the opposite of the response necessary to maintain fluidity at low temperatures. The change in branched relative to straight-chain fatty acids is small because there is also an increase in $a 16: 0$ at lower temperatures. Even the small amount of 16:1 changes in the "wrong" direction to be part of an adaptive mechanism at low temperatures. This pattern of adaptation is completely different to that reported by Russell \& Fukunaga (1990) for another psychrotolerant Bacillus sp. and by Suutari \& Laakso (1992) for mesophilic Bacillus subtilis.
Another complexity of the fatty acid changes is that they are not always linear with temperature, i.e. there may be different adaptations at the upper and lower ends of the growth temperature range. Such a bimodal thermal adaptation is seen in Arthrobacter sp. C2-2. When growth temperature is lowered from 25 to $15^{\circ} \mathrm{C}$ there is little change in the proportion of branched-chain fatty acids because the increase in $a 15: 0$ is matched by a decrease in $a$ 1 7:0 and, since there are few other compositional changes, the main fluidity-modulating factor is a shortening of the fatty acyl chain length. When the temperature is lowered further to $5^{\circ} \mathrm{C}$ there are further changes in $a 15: 0$ and $a 17: 0$ but in addition there is a decrease in $n 17: 0$. Overall this results in an increase in the ratio of branched relative to straight-chain fatty acids and the proportion of anteiso-relative to iso-branching, as well as the change in chain length. Looked at across the growth temperature range, as temperature is lowered the ratio of branched to straight-chain fatty acids and the acyl chain length increase and decrease respectively in a linear fashion, whereas the ratio of anteiso-to iso-branching decreases and then increases.

In conclusion, we have found that there is no single strategy of thermal adaptation of lipid composition that is used by the dominant culturable bacteria in an Antarctic soil. Instead, a variety of strategies are used, which do not follow phylogenetic boundaries and which show a great diversity and complexity. Moreover, we have found some changes which have not been reported previously. Whether they represent specific adaptations to the particular cold environment of Antarctic soil will require further investigation of both Antarctic and other cold habitats, such as permanently cold Alpine soils.

\section{Acknowledgements}

This research was carried out with the support of a grant from the Natural Environment Research Council. We are grateful to Professor L. Finegold for his refereeing comments on the manuscript, and to an anonymous referee who pointed out our error in presentation of specific growth rates.

\section{References}

Bhakoo, M. \& Herbert, R.A. 1980. The fatty acid and phospholipid composition of five psychrotrophic Pseudomonas spp. grown at different temperatures. Archives of Microbiology, 126, 51-55

Boggs, J.M., Stamp, D. \& Moscarello, M.A. 1981. Interaction of myelin basic-protein with dipalmitoylphosphatidylglyceroldependence on the lipid phase and investigation of the metastable state. Biochemistry, 20, 6066-6072

Canillac, N., Pommier, M.T. \& Gounot, A.M. 1982. Effet de la temperature d'incubation sur la composition lipidique de Corynebacteriacees du genre Arthrobacter. Canadian Journal of Microbiology, 28, 284-290.

Friedmann, E.I. 1993. ed. Antarctic microbiology. New York: WileyLiss, $684 \mathrm{pp}$.

Harwood, J.L. \& Russell, N.J. 1984. Lipids of plants and microbes London: George Allen \& Unwin, 162 pp 
Kates, M. 1986. Techniques of lipidology. Amsterdam: Elsevier, $464 \mathrm{pp}$.

MoRita, R.Y. 1975. Psychrophilic bacteria. Bacteriological Reviews, 39, 144-167.

Russell, N.J. 1984. Mechanisms of thermal adaptation in bacteria: blueprints for survival. Trends in Biochemical Sciences, 9, 108112.

RUSSELL, N.J. 1989. Functions of lipids: structural roles and membrane functions. In RatLedGe, C. \& Wilkinson, S.A., eds. Microbial lipids. London: Academic Press, 279-365.

Russell, N.J. 1990. Cold adaptations of microorganisms. Philosophical Transactions of The Royal Society of London, B326, 595-611.

Russell, N.J. \& FukunaGa, N. 1990. A comparison of thermal adaptations of membrane lipids in psychrophilic and thermophilic bacteria. FEMS Microbiology Reviews, 75, 171-182.

SUUTARI, M. \& LAAKSO, S. 1992. Unsaturated and branched chain fatty acids in temperature adaptation of Bacillus subtilis and Bacillus megaterium. Biochimica et Biophysica Acta, 1126, 119-124.
TORSVIK, V., SORHEIM, R. \& GoKsøYR, J. 1996. Total bacterial diversity in soil and sediment communities: a review. Journal of Industrial Microbiology, 17, 170-178.

VINCENT, W.F. 1988. Microbial ecosystems of Antarctica. Cambridge: Cambridge University Press, $304 \mathrm{pp}$.

Wada, M., Fununaga, N. \& SaSaKi, S. 1984. Effect of growth temperature on phospholipid fatty acid composition in a psychrotrophic Pseudomonas sp. strain E3. Plant Cell Physiology, 28, 1209-1217.

WhITE, P.L. 1999. The effects of environmental warming on Antarctic soil microbial communities. $\mathrm{PhD}$ thesis, University of London, $\mathrm{UK}$, $230 \mathrm{pp}$. [Unpublished.]

WYNN-Williams, D.D. 1990. Ecological aspects of Antaretic microbiology. Advances in Microbial Ecology, 11, 71-146. 